\title{
Editorial
}

\section{Infarto agudo al miocardio asociado al uso de cocaína ¿una entidad diferente?}

\author{
Alberto Fuenzalida ${ }^{1-2}$, Martín Valdebenito ${ }^{1}$, Alejandro Fajuri ${ }^{1}$ \\ 1. Departamento Enfermedades Cardiovasculares, Pontificia Universidad Católica de Chile, Santiago. \\ 2. Residente de Cardiología, Pontificia Universidad Católica de Chile
}

El interesante trabajo presentado por Veas et $\mathrm{al}^{1}$ en este número de la revista genera ciertas interrogantes sobre la fisiopatología y manejo de aquellos pacientes que están cursando un infarto agudo al miocardio con supradesnivel del ST y que refieren consumo reciente de cocaína.

Correspondencia:

Dr. Alejandro Fajuri

División de Enfermedades Cardiovasculares

Facultad de Medicina, Pontificia Universidad Católica de Chile

Marcoleta $367,8^{\circ}$ piso.

Santiago, Chile. CP 8330024

afajuri@puc.cl 
Es sabido que la cocaína es una de las drogas más usadas a nivel mundial, alcanzando un $0,4 \%$ de prevalencia anual, siendo mayor su consumo en Norteamérica y Sudamérica. En Chile, según datos del Servicio Nacional para la Prevención y Rehabilitación del Consumo de Drogas y Alcohol (SENDA), el consumo llega a un 1,1\% al año, manteniéndose relativamente estable ${ }^{2}$.

El dolor torácico es una de las causas más frecuentes de consulta en los servicio de urgencia. Los pacientes que han consumido cocaína y que ingresan por dolor torácico tienen más frecuentemente infarto agudo al miocardio con elevación del ST, así como también otros síndromes coronarios ${ }^{3}$. Del total de infartos agudos, las cifras de aquellos con consumo reciente de cocaína varían desde $0,9 \%$ en un registro estadounidense referido con autoreporte ${ }^{4}$, hasta un $17 \%$ cuando se utiliza un medio analítico dirigido. El presente estudio de Veas et al ${ }^{1}$ reporta un 4,5\% validado con autoreporte. Los pacientes consumidores de cocaína usualmente subreportan el consumo desde un 20 a un 50\% 5-3. Se han establecido diversos mecanismos por los que se producen infartos del miocardio asociados al consumo de cocaína (IAMC) ${ }^{6-7}$. Estos incluyen activación simpaticomimética, vasoespasmo coronario, disfunción endotelial, trombosis y ateroesclerosis acelerada. La activación simpaticomimética propia de la cocaína conlleva un efecto inotrópico y cronotrópico positivo, aumentando además la presión arterial, todo lo cual conlleva a un aumento del stress de la pared del ventrículo izquierdo y a un mayor consumo de oxígeno; este mecanismo por sí sólo es incapaz de explicar la presencia de un infarto con elevación del ST. El vasoespasmo se ha descrito como consecuencia del consumo de cocaína ${ }^{8}$, pero reportes clínicos de pacientes con IAMC no han podido demostrar este efecto ${ }^{3}$. Los pacientes consumidores poseen marcadores de actividad plaquetaria aumentada ${ }^{9}$. Esta activación plaquetaria estaría dada por disfunción endotelial más que por un efecto directo, donde la cocaína y sus metabolitos producen secreción endotelial de factor Von Willebrand $\left(\mathrm{fVW}^{9}\right)$, el que tendría directa relación con la formación de puentes plaquetarios; este efecto es más marcado en arteriolas cerebrales que en territorio coronario ${ }^{10}$. Esta mayor secreción de fVW se da en territorios arteriales más pequeños donde el stress de la pared es mayor, con un posible mayor efecto en la microcirculación. La disfunción endotelial juega un rol predominante. Se han descrito múltiples marcadores de disfunción endotelial que persisten incluso al suspender el consumo $^{11}$, existiendo alteración de la producción de óxido nítrico y de la actividad de la óxido nítrico sintetasa (eNOS) con predisposición a mayor infiltración leucoci- taria ${ }^{12}$, aumentando así el factor tisular y disminuyendo la expresión del inhibidor del factor tisular ${ }^{13}$. Todo lo anterior predispone a arterioesclerosis acelerada y un estado protrombótico.

Desde el punto de vista angiográfico, los IAMC tienen mayor reporte de arterias coronarias sin lesiones significativas que van desde un 25 a un 40\%, menos lesiones de 3 vasos $^{4}$, y más incidencia de trombos ${ }^{3-14}$. Existiría una enfermedad ateroesclerótica más difusa con más cantidad de placas, pero estenosis menos severas ${ }^{15}$. Estudios postmortem encuentran lesiones en vaso de pequeño calibre con hipertrofia de la media, proliferación de íntima y fibrosis periadventicial ${ }^{16}$. Algunos reportes con ecografía intracoronaria (IVUS) demuestra mayor cantidad de placa excéntrica fibrosa ${ }^{17}$. Pacientes usuarios de cocaína presentan mayor alteración del flujo microvascular. Esto se ha evidenciado con el TIMI Frame Count (cTFC) donde, a pesar de presentar arterias coronarias sin lesiones significativas y buen flujo epicárdico, presentan peor flujo microvascular ${ }^{18-19}$. El trabajo presentado por Veas et al ${ }^{1}$ evidencia el mismo fenómeno con alteración de la microvasculatura representada por el cTFC con una alteración que persiste, incluso, posterior al tratamiento invasivo.

Llama la atención que a pesar de reportar una alta tasa de trombos la aspiración y la trombolisis intracoronaria han sido subutilizadas. Es así como hay reportes exitosos de trombolisis intracoronaria ${ }^{20-21-22}$ e inhibidores IIb/IIIa ${ }^{23}$. La experiencia presentada por Veas et $\mathrm{al}^{1}$ tiene una menor tasa de uso de stent y dilataciones, presumiblemente explicado por el buen flujo de las arterias epicárdicas.

Todo lo anteriormente expuesto, sobretodo el acentuado fenómeno de trombosis y disfunción endotelial con una mayor repercusión a nivel microvascular, pone de manifiesto una serie de dudas con respecto a la fisiopatología y a la manera de enfrentar en el pabellón de hemodinamia a los pacientes con un infarto con supradesnivel del ST y antecedente de consumo reciente de cocaína .

1) ¿Es de utilidad caracterizar las placas ateroescleróticas en estos pacientes con herramientas como el IVUS con Chromaflow o la Tomografía de coherencia óptica (OCT)?; 2) ¿Se beneficiarían de trombolísis intracoronaria?, ¿tendrán diferentes efectos los distintos antiagregantes? 3) ¿Las alternativas deben ir dirigidas a mejorar el flujo microvascular más que el flujo de arterias epicárdicas?.

Creemos que es necesario mejorar la evaluación de estas variables y así incorporarla a nuevos protocolos de investigación. 


\section{Referencias}

1. VEAS N, LINDEFJELD DS, WINTER JL, MÉNDEZ M, PÉREZ O, MÁRTINEZ A, et al. Deterioro del flujo microvascular coronario durante la angioplastía primaria en pacientes consumidores de cocaína. Rev Chil Cardiol 2014; 33: 123-126

2. SENDA.GOBIERNO DE CHILE. Noveno estudio nacional de drogas en población general en Chile. Disponible en http:// www.senda.gob.cl

3 BOSCH X, LOMA-OSORIO P, GUASCH E, NOGUE S, ORTIZ JT, SANCHEZ M. Prevalence, clinical characteristics and risk of myocardial infarction in patients with cocaine-related chest pain. Rev Esp Cardiol 2010; 63: 1028-1034

4 GUPTA N, WASHAM JB, MOUNTANTONAKIS S E, LI S, ROE MT, DE LEMOS JA, et al. Characteristics, management, and outcomes of cocaine-positive patients with acute coronary syndrome (from the National Cardiovascular Data Registry). Am J Cardiol 2014; 113: 749-756

4 HOLLANDER JE, TODD KH, GREEN G, HEILPERN KL, KARRAS DJ, SINGER AJ, et al. Chest pain associated with cocaine: an assessment of prevalence in suburban and urban emergency departments. Ann Emerg Med 1995;26: 671-6.

5. MARAJ S, FIGUEREDO VM, LYNN MORRIS D. Cocaine and the heart. Clin Cardiol 2010; 33: 264-269

6. MCCORD J, JNEID H, HOLLANDER JE, DE LEMOS JA, CERCEK B, HSUE P, et al. Management of cocaine-associated chest pain and myocardial infarction: a scientific statement from the American Heart Association Acute Cardiac Care Committee of the Council on Clinical Cardiology. Circulation 2008; 117:1897-1907

7. GURUDEVAN SV, NELSON MD, RADER F, TANG X, LEWIS $\mathrm{J}$, JOHANNES J, et al. Cocaine-induced vasoconstriction in the human coronary microcirculation: new evidence from myocar- dial contrast echocardiography. Circulation 2013; 128:598-604

8. PEREIRA J, SAEZ CG, PALLAVICINI J, PANES O, PEREIRA-FLORES K, CABRERAS M J, et al. Platelet activation in chronic cocaine users: effect of short term abstinence. Platelets 2011; 22: 596-601

9. HOBBS WE, MOORE EE, PENKALA RA, BOLGIANO DD, LOPEZ JA. Cocaine and specific cocaine metabolites induce von Willebrand factor release from endothelial cells in a tissue-specific manner. Arterioscler Thromb Vasc Biol 2013; 33: $1230-1237$

10. SAEZ CG, OLIVARES P, PALLAVICINI J, PANES O, MORENO N, MASSARDO T, et al. Increased number of circulating endothelial cells and plasma markers of endothelial damage in chronic cocaine users. Thromb Res 2011; 128: e18-23

11. PRADHAN L, MONDAL D, CHANDRA S, ALI M, AGRAWAL KC. Molecular analysis of cocaine-induced endothelial dysfunction: role of endothelin-1 and nitric oxide. Cardiovasc Toxicol 2008; 8: 161-171

12. STEFFEL J, ISELI S, ARNET C, LUSCHER TF, TANNER FC. Cocaine unbalances endothelial tissue factor and tissue factor pathway inhibitor expression. J Mol Cell Cardiol 2006; 40: 746-749

13. MÉNDEZ M, MARTÍNEZ G, VEAS N, PÉREZ O, LINDEFJELD D, WINTER JL, et al. Infarto Agudo al Miocardio en pacientes menores de 40 años. Características clínicas, angiográficas y alternativas terapéuticas. Rev Chil Cardiol 2013; 32: $21-27$

14. EBERSBERGER U, SUDARSKI S, SCHOEPF U J, BAMBERG F, TRICARICO F, APFALTRER P, et al. Atherosclerotic plaque burden in cocaine users with acute chest pain: analysis by coronary computed tomography angiography. Atherosclero- 
sis $2013 ; 229: 443-448$

15. SCHWARTZ BG, REZKALLA S, KLONER RA. Cardiovascular effects of cocaine. Circulation 2010;122: 2558-2569

16. SONNE C, STEMPFLE HU, KLAUSS V, SCHIELE TM. Intravascular ultrasound-guided percutaneous coronary intervention in a human immunodeficiency virus-positive patient with cocaine-associated acute myocardial infarction: case report and review. Heart Lung Circ 2005; 14: 197-200

17. TURHAN H, AKSOY Y, OZGUN TEKIN G, YETKIN E. Cocaine-induced acute myocardial infarction in young individuals with otherwise normal coronary risk profile: is coronary microvascular dysfunction one of the underlying mechanisms? Int $\mathbf{J}$ Cardiol 2007; 114: 106-107

18. WEBER JE, HOLLANDER JE, MURPHY SA, BRAUNWALD E, GIBSON CM. Quantitative comparison of coronary artery flow and myocardial perfusion in patients with acute myocardial infarction in the presence and absence of recent cocaine use. J Thromb Thrombolysis 2002; 14: 239-245

19. VILLOTA JN, RUBIO LF, FORES JS, PERIS VB, BURGUERA EP, GONZALEZ VB, et al. Cocaine-induced coronary thrombosis and acute myocardial infarction. Int J Cardiol 2004; 96: 481-482

20. YAO SS, SPINDOLA-FRANCO H, MENEGUS M, GREENBERG M, GOLDBERGER, M, SHIRANI J. Successful intracoronary thrombolysis in cocaine-associated acute myocardial infarction. Cathet Cardiovasc Diagn 1997; 42: 294-297

21. HADJIMILTIADES S, COVALESKY V, MANNO BV, HAAZ WS, MINTZ GS. Coronary arteriographic findings in cocaine abuse-induced myocardial infarction. Cathet Cardiovasc Diagn 1988; 14: 33-36

22. FRANGOGIANNIS NG, FARMER JA, LAKKIS NM. Tirofiban for cocaine-induced coronary artery thrombosis: a novel therapeutic approach. Circulation 1999; 100:1939. 